\title{
LA ACTITUD LINGUÍSTICA EN LA COMUNIDAD DE HABLA DE MAGALLANES: APROXIMACIÓN A SUS COMPONENTES BÁSICOS ${ }^{1}$
}

MARGARITA MAKUC

\section{RESUMEN}

El lenguaje hablado de la región de Magallanes reúne un conjunto de características que le dan singularidad cultural al hablante de la región. Es importante destacar que la geografía, la actividad ganadera, el clima, entre otros factores contextuales, que rodean al hombre en la Patagonia austral (Martinic, 2006) se manifiestan de diversos modos en la variación dialectal de la región, la que ha conservado rasgos lingüísticos a partir de su condición de aislamiento geográfico (Makuc, 2010a , Martinic y Buratovic, 2007). La variación dialectal constituye un aspecto identificado en diversos estudios (Oroz, 1965, Araya, 1973, Makuc, 1999, 2008, 2010ª). Un aspecto relevante en la conservación de variantes lingüísticas obedece a una actitud lingüística favorable que caracteriza a la comunidad de habla magallánica y que permite que las variantes en los diversos niveles de la lengua se conserven y sobrevivan en un mundo cada vez más homogeneizante (Hudson, 1981, López Morales, 1989, 1994; Silva, 1989; Blas, J. 1999). El estudio de la actitud de los hablantes hacia las diversas manifestaciones de la variación del la lengua se ha abordado a partir de los datos obtenidos en las ciudades de Punta Arenas, Puerto Natales y la Isla de Tierra del Fuego en el marco de la Encuesta de Identidad Regional elaborada para el estudio de identidad ${ }^{2}$ (Makuc, 2010 a). Los resultados evidencian el predominio de una actitud positiva de los hablantes hacia los usos del español en la región, es ampliamente reconocido y aceptado el hecho de que los magallánicos tienen una particular forma de hablar así como un acento que los distingue del resto de los habitantes del país, estas variaciones del español estándar son valoradas positivamente y como un rasgo que fortalece la identidad de los habitantes.

PALABRAS CLAVE: variación, dialecto, actitud lingüística.

Universidad de Magallanes, Facultad de Humanidades, Cienciasa Sociales y de la Salud. Av. Bulnes 01855, Punta Arenas, Chile.margarita.makuc@umag.cl.

1 Estudio sociolingüístico de la variación dialectal ejecutado en el marco del Programa de investigación (PR-F4-01EH-09-10) financiado por la Dirección de Investigación de la Universidad de Magallanes.

2 Estudio para el fortalecimiento de la Identidad regional, financiado por el gobierno regional de Magallanes y Antártica Chilena y la subsecretaría de desarrollo Regional (SUBDERE). 


\title{
LINGUISTIC ATTITUDE IN THE SPEAKING COMMUNITY OF MAGALLANES BASIC COMPONENTS APROACH
}

\begin{abstract}
The spoken language of the region of Magallanes gathers a series of characteristics which give the regional speaker a cultural singularity. It is important to highlight that geography, cattle raising activity and weather, among other contextual factors that surround the people in the southern patagonia (Martinic, 2006), are manifested in different ways in the dialectal variation of the region, which has preserved linguistic features from its geographical isolation condition (Makuc, 2010a, Martinic y Buratovic, 2007). Dialectal variation constitutes an aspect identified in different studies (Oroz, 1965, Araya, 1973, Makuc, $1999,2008,2010^{\text {a)}}$. A relevant aspect in the conservation of linguistic variants is due to a favorable linguistic attitude which characterizes the Magellan speaking community and allows that the variants at different levels of the language stay and survive in a world increasingly homogenizing. (Hudson, 1981, López Morales, 1989, 1994; Silva, 1989; Blas, J. 1999) The study about he attitude of the speakers towards the various manifestations of the variation of the language has been carried out from the data obtained in the cities of Punta Arenas, Puerto Natales and Tierra del Fuego island in the framework of the Regional Identity Survey developed for the study of identity[2] (Makuc, 2010). The results show the prevalence of a positive attitude of the speakers towards the uses of Spanish in the region, it is highly recognized and accepted the fact that Magellan people have a particular way of speaking as well as an accent that distinguishes them from the rest of the inhabitants of the country, these variations of standard Spanish are valued positively and as a feature that strengthens the identity of the inhabitants.
\end{abstract}

KEY WORDS: variation, dialect, linguistic attitude.

\section{INTRODUCCIÓN}

El estudio del español de Magallanes ha permitido observar elementos lingüísticos propios de la región cuya presencia se explicaría por la conjunción de factores geográficos, históricos y de poblamiento; diversos autores (Oroz, 1965; Kany, 1970; Araya, 1973) plantean que el español de Magallanes manifiesta gran influencia de la isla de Chiloé (Makuc, 1999, 2008). Al mismo tiempo, es posible observar la influencia de los habitantes de la Patagonia argentina en el nivel fonológico y léxico, así como la presencia de extranjerismos que en el nivel léxico han dado origen a nuevos términos (guachimán, yam de ruibarbo). En este estudio interesa abordar la actitud lingüística como un elemento que contribuye a reforzar la permanencia de variantes lingüísticas identificadas en el marco de investigaciones sociolingüísticas previas orientadas a la caracterización de la comunidad de habla de Magallanes. Dichos estudios se han realizado a partir de una serie de manifestaciones lingüísticas propias de la interacción comunicativa en el contexto regional determinando las variaciones de la lengua en sus diversos niveles: fonológico, morfológico, sintáctico-semántico, léxico y textual (Makuc, 1999, 2008, 2010).

\section{1.- LA VARIACIÓN LINGÜÍSTICA EN LA REGIÓN DE MAGALLANES}

El conjunto de características del lenguaje hablado en la región de Magallanes nos permite hablar de variaciones sociolingüísticas que le dan singularidad cultural a la comunidad de habla de Magallanes en la cual se observan particularidades registradas en diversos estudios (Oroz, 1965; Araya, 1973; Makuc, 2010 a y b). La variación se plantea en los diversos niveles de la lengua: nivel fonológico, morfológico, sintáctico-semántico, léxico y textual. En el nivel fonológico un rasgo que representa la singularidad del habla es la ausencia de variantes fonológicas socialmente estigmatizadas características del habla de baja escolaridad (Silva, 1989), igualmente la fricativización del fonema /ch/ ha sido registrada por diversos autores (Oroz, 1965; Guitarte, 1991). Por su parte, en el nivel morfológico se destaca la 
absoluta supresión en el habla de pronombres clíticos reduplicados Silva (1982) esta ausencia evidenciaría un proceso de control de expansión de variantes estigmatizadas (Almeida, 2003). La variación sintáctica, en tanto se ha abordado en el estudio de la variación verbal, la cual ha sido analizada por diversos autores que registran el uso causativo del verbo como una variante del sur de Chile, específicamente en Magallanes (Punta Arenas, Puerto Natales y Porvenir). Estudios recientes señalan que este uso reflejaría un proceso de ergativización, (Mendikoetxea, 1999; Demonte, 2000; Sabaj, 2002; Makuc, 2008) que implica, en términos generales, la explicitación del agente de la acción expresada en el verbo. Proceso similar se observa en lo que respecta a la variación léxica, en la comunidad de habla magallánica se presentan una serie de expresiones reconocidas asociadas a la actividad ganadera y económica (tumbear-carnaso-changarín guachimán bagual, gomería) a la geografía (escarcha negra, agua nieve) a la alimentación (churrasquear reitimiento, baguet- chulengueada-chulengo). Diversos autores (Coloane, 1956, 1983, 2002; Mihovilovic, 1974; Moreno, 2007; Vega, 2001; Wegmann, 1976; Makuc, 2010b) ha registrado este tipo de variación léxica. Finalmente, en el nivel textual se observan manifestaciones que distinguen a la comunidad de habla en estudio, tal es el caso de los Los Mensajes para el campo (Martinic y Buratovic, 2007; Makuc, 2010 ${ }^{\mathrm{a}}$ ) que constituyen un elemento comunicativo identitario de la comunidad de habla magallánica, estos mensajes presentan particularidades sintácticas, léxicas y textuales que actualizan elementos discursivos propios del contexto de situación en que se producen, pues forman parte de espacios radiales que conservan en la actualidad textos tal cual fueron creados a principios de siglo (Makuc, 2010b).

\section{2.- LA ACTITUD LINGÜÍSTICA Y LA VARIACIÓN}

El estudio de la actitud lingüística se enmarca en el concepto de "comunidad de habla", esta noción para diversos autores, no sólo incluye a un grupo de hablantes que usa las mismas formas lingüísticas, sino que principalmente comparte las mismas normas con respecto a una lengua (Labov, 1983; Silva, 1989). El conjunto de hablantes que comparte (además de una lengua) un conjunto de normas y valores de naturaleza sociolingüística pertenece a una comunidad de habla, pues comparten actitudes lingüísticas, reglas de uso y son capaces de reconocerse como integrantes de esta comunidad. Por lo anterior los miembros de una comunidad de habla suelen acomodar su discurso a las normas y valores compartidos, por ello, el cumplimiento de las normas sociolingüísticas al que obliga la pertenencia a una comunidad puede servir de marca diferenciadora, de marca de grupo (López Morales, 1989). Diversos autores (Fasold, 1984; López Morales, 1989; Almeida y Vidal, 1995) destacan la importancia del estudio de la actitud, en tanto ésta contribuye a la difusión de los cambios lingüísticos, evidenciando patrones de uso, así como la evaluación de los hablantes de la variación lingüística. En general, la noción de actitud se ha definido como una disposición a reaccionar favorable o desfavorablemente hacia una serie de objetos, en términos lingüísticos podemos hablar de una postura crítica, valorativa de los hablantes hacia fenómenos específicos de la lengua (Fasold, 1984). Existe acuerdo en que las actitudes constituyen una predisposición a la acción, que es aprendida y susceptible de modificarse a pesar de su relativa estabilidad (Agheyisi y Fishman, 1970). Para un amplio sector de investigadores la actitud lingüística integra tres componentes básicos(a) ideológicos (conjunto de creencias, ideas y convicciones), (b) afectivos (sentimientos favorables o desfavorables) y (c) de comportamiento: tendencia a pensar y actuar de modo congruente con los sentimientos e ideas en diversas situaciones sociales. La actitud lingüística estaría dominada por el rasgo conativo, pues las creencias junto al saber proporcionado por la conciencia provocarían determinadas actitudes que, finalmente, afectarían la actuación lingüística (Almeida y Vidal, 1995). Respecto a su origen, diversos estudios (López Morales, 1989; Almeida y Vidal, 1995) han demostrado que las diferencias lingüísticas o estéticas no explican el origen de las actitudes lingüísticas, sino convenciones relacionadas con el estatus y el prestigio asociado a las personas que hablan dicha lengua o variedad de lengua, desde esta perspectiva, la evaluación positiva o negativa se debería a apreciaciones subjetivas, en donde la consideración es principalmente sociocultural. Una corriente importante de la investigación sociolingüística y que se vincula estrechamente con nuestro estudio, ha planteado la relación entre lenguaje e identidad 
de los individuos, relación que halla su expresión más directa en las actitudes de los individuos hacia las lenguas y sus usuarios, desde esta perspectiva se propone la noción de "acto de identidad" como una forma de explicar el hecho de que los hablantes crean sus reglas lingüísticas para parecerse a aquellos grupos con los que quieren identificarse. En el mismo sentido se orientan los estudios sobre actitudes hacia la variación regional (Ortega, 1981; Blas, 1996, 1999) los cuales han mostrado la evaluación que realizan los hablantes de su propia variedad regional, entre los resultados obtenidos se observa que dichos hablantes otorgan mayoritariamente un valor más alto a la variante estándar que a su propia variedad (local, regional). Siguiendo este enfoque, el estudio de la actitud lingüística de los hablantes magallánicos resulta relevante en la caracterización de la identidad de los magallánicos, por lo que los resultados de este estudio forman parte de la caracterización de aspectos centrales de la identidad de los habitantes de la región.

\section{METODOLOGÍA Y PROCEDIMIENTOS DEL ESTUDIO}

Las metodologías de la investigación sociolingüística (López, 1994; Almeida y Díaz, 1998, Almeida, 2003) se han aplicado al diseño de la Encuesta de Identidad Regional, la cual fue administrada a una muestra de 1488 personas adultas de 18 o más años de edad, con residencia permanente en la región de Magallanes y Antártica Chilena. Dicha encuesta, de representatividad regional y provincial fue aplicada en ciudades de la región. Desde un punto de vista metodológico, el estudio se define como de tipo exploratorio-descriptivo y se orientó a describir aspectos generales de la actitud lingüística respecto del español de Punta Arenas, entendido éste como una variante dialectal del español/castellano (Silva, 1982; Moreno, 1990; López, 1994; Almeyda, 2003, Makuc, 2010). Los objetivos del estudio se orientan a caracterizar la actitud lingüística en los hablantes de Magallanes identificando los componentes ideológicos, afectivos y de comportamiento explicitados en las preguntas relacionadas con el uso de la lengua.Para definir el universo del presente estudio, se tomó como base el Marco muestral utilizado para el Programa Integrado de Encuestas de Hogares (PIDEH), el cual divide la estratos y éstos, en secciones o conglomerados geográficos, con información sobre viviendas y población obtenida del Censo de Población y Viviendas 2002. La población objetivo es la que habita en el territorio continental la Región de Magallanes y comprende a las personas con edades de 18 o más años de edad, que residen habitualmente en viviendas particulares seleccionadas.

\section{PRESENTACIÓN Y DISCUSIÓN DE RESULTADOS}

A continuación se presentan los resultados organizados en los componentes descritos para abordar la actitud lingüística; el primero de ellos (componente ideológico) describe la identificación de los hablantes con la comunidad de habla magallánica y la consideración de ésta como elemento distintivo del resto de hablantes del país. El segundo componente (afectivo) caracteriza la valoración y evaluación de las variantes dialectales de la comunidad de habla; finalmente, el componente del comportamiento se analiza a partir del tópico que hemos caracterizado como "reconocimiento y uso de elementos lingüísticos propios de la comunidad de habla magallánica en el nivel fonológico y léxico de la lengua.

a) Componente ideológico: conjunto

de creencias, ideas y convicciones

Para abordar el componente ideológico de la actitud lingüística de los hablantes se ha propuesto el tópico que hemos definido como: "Identificación con la comunidad de habla magallánica como elemento distintivo del resto de hablantes del país". En este tópico, los resultados evidencian una clara conciencia de pertenencia a una comunidad de habla que presenta diferencias con la norma estándar. Al respecto, los hablantes encuestados señalan sentirse muy identificados con la forma de hablar de los habitantes de la región (54\%),por el contrario un porcentaje menor $(9,2 \%)$ señala que no se identifica con la forma de hablar de los habitantes de la región. Por otra parte, frente a la pregunta: ¿Existen diferencias en la forma de hablar con el resto de los habitantes de Chile? el 70,6\% de ellos señala que efectivamente existen diferencias, en tanto sólo el $26,3 \%$ de los encuestados señala que no existen tales diferencias. Como una forma de complementar esta información 
se elaboró una pregunta que permitiera observar el grado de cercanía e identificación entre hablantes de tres ciudades de la región de Magallanes (Punta arenas, Puerto Natales y Porvenir). Al respecto los resultados obtenidos permiten señalar que del total de los encuestados, el 75,1\% de ellos señala que no existen diferencias en la forma de hablar entre personas que viven en Puerto Natales, Porvenir y en Punta Arenas. Mientras que sólo el 14,7\% señala que sí existen estas diferencias.

b) Componente afectivo: sentimientos

favorables o desfavorables hacia la

variedad de lengua de los hablantes

En este componente se estudia la valoración y evaluación de las variantes dialectales de la comunidad de habla, ésta se abordó a partir de tres preguntas, cuyos resultados se presentan a continuación. En la primera pregunta referida a los lugares donde se hablaría mejor el castellano los encuestados expresan que en Magallanes se habla mejor el castellano (44,9\%); al contrario un reducido porcentaje de entrevistados (5.9\%) considera que el castellano se habla mejor en otras ciudades del país. Un resultado similar se observa en la pregunta ¿Que tan de acuerdo está con la expresión: En Magallanes se habla "mejor" que en el resto de Chile. Los resultados obtenidos permiten señalar que del total de los encuestados, el $69.2 \%$ de los habitantes de la región señala compartir la expresión; en tanto el 30,8\% de los encuestados difiere de esta afirmación. En la tercera pregunta referida a la valoración se plantea: ¿Que tan de acuerdo está con la expresión: Los magallánicos hablan "peor" que el resto de los habitantes de Chile, observamos que sólo un $15 \%$ de los encuestados señala estar de acuerdo con la afirmación; por otra parte, un porcentaje importante $(85,1 \%)$ no comparte dicha afirmación. Por otra parte, el 6,5\% de los habitantes de la región señala estar de acuerdo con la expresión: Los magallánicos hablan peor que el resto de los habitantes de Chile. Por su parte, el 53,1\% de los encuestados señala estar en desacuerdo con la expresión: Los magallánicos hablan peor que el resto de los habitantes de los encuestados señala estar muy en desacuerdo con la expresión antes mencionada. Como podemos observar, comparativamente la región es percibida como un lugar en donde el español se habla mejor que en el resto del país, es posible señalar que en términos valorativos, de acuerdo a estos datos, los hablantes de la región evidencian una actitud positiva frente a la variedad regional.

c) Componente de comportamiento: tendencia a pensar y actuar de modo congruente con los sentimientos e ideas en diversas situaciones sociales

Este componente se analiza a partir del tópico que hemos caracterizado como "reconocimiento y uso de elementos lingüísticos propios de la comunidad de habla magallánica en el nivel fonológico y léxico de la lengua. Como una forma de abordar el reconocimiento de particularidades lingüísticas percibidas por los hablantes como propias de la comunidad de habla en estudio, se plantearon dos preguntas que abordan el componente fonético y léxico respectivamente; la primera pregunta plantea a los encuestados: "Qué tan de acuerdo está con la expresión: las personas que viven en la región tiene un acento que los distingue del resto", la respuesta a esta pregunta evidencia que el 91.9\%, ratifica esta afirmación; mientras que sólo el $8,1 \%$ no percibe

Tabla 1. Síntesis de resultados obtenidos en el componente ideológico, afectivo y de comportamiento de la actitud lingüística de hablantes magallánicos.

\begin{tabular}{|l|c|c|}
\hline $\begin{array}{l}\text { COMPONENTE } \\
\text { IDEOLÓGICO }\end{array}$ & IDENTIFICACIÓN CON LA & $\begin{array}{c}\text { RECONOCIMIENTO DE DIFERENCIAS } \\
\text { CON EL RESTO DEL PAÍS } \\
\text { COMPONE HABLAR }\end{array}$ \\
\hline $\begin{array}{l}\text { COMECTIVO } \\
\text { AFE }\end{array}$ & $54 \%$ & $70,6 \%$ \\
\hline COMPONENTE & VALORACIÓN POSITIVA DEL & VALORACIÓN NEGATIVA DEL \\
COMPORTAMIENTO & HABLA MAGALLÁNICA & HABLA MAGALLÁNICA \\
& $56,8 \%$ & $15 \%$ \\
\hline
\end{tabular}


esta diferencia. En lo relativo al componente léxico, los resultados permiten señalar que del total de los encuestados, el 92,3\% de los habitantes de la región reconoce y menciona elementos léxicos que considera propios de la comunidad de habla magallánica.

El análisis global de la actitud lingüística de los hablantes magallánicos nos permite identificar en los tres componentes centrales de este concepto el predominio de una actitud positiva de los hablantes hacia los usos del español en la región. En el componente ideológico un porcentaje importante de hablantes concuerda en cuanto a que pertenecen a una comunidad de habla que tiene diferencias con el resto del país. En cuanto al componente afectivo, los hablantes de la región valoran en su mayoría positivamente el español que se habla en Magallanes; así como es reconocido que los magallánicos tienen una particular forma de hablar, así como elementos léxicos distintivos de la variación dialectal.

\section{CONCLUSIONES GENERALES}

La comunidad de habla magallánica, presenta un alto grado de cohesión en cuanto a la validez de normas y valores sociales, por lo que podemos hablar de una actitud compartida por hablantes de las ciudades principales convirtiéndose en una realidad regional. Este estudio de la actitud lingüística proporciona elementos de juicio que nos permiten dar una respuesta respecto de los límites de la comunidad lingüística en cuanto al modo y el grado en que diversas zonas comparten normas y valores sociales, de este modo esta comunidad de habla se constituye en una comunidad de consenso, de sintonía entre grupos e individuos diferentes, donde el conflicto estaría minimizado. El análisis de los resultados nos permite inferir la existencia de una idea de pertenecía asociada a la lengua en uso y al mismo tiempo al reconocimiento de diferencias con el resto del país, estas diferencias, además, son valoradas positivamente en términos de los aspectos fonológicos y léxicos, por otra parte, el habla de otras regiones no es considerada como la variante estándar, observándose una desfavorable actitud hacia la forma de hablar de habitantes del resto de Chile. En este sentido comunidad de habla y actitud se interrelacionan para explicar las variaciones lingüísticas, en tanto ellas nos permiten recoger información acerca de los hablantes, el predominio de una actitud favorable respecto de la variación es un indicio del futuro de fenómenos variables en cuanto a la posibilidad de que algunas de las variantes se conviertan en la norma categórica. En términos generales las variantes lingüísticas se han conservado no sólo por particularidades sociales y geográficas, sino además por aspectos que dicen relación con una actitud lingüística positiva, factor que explicaría, en conjunto con los anteriores, la permanencia de variantes patrimoniales en el habla de Magallanes.

\section{BIBLIOGRAFÍA}

AGHEYISI, R. y J. FISHMAN 1970. Language attitude studies: A brief survey of methodological approaches. Antrophological Linguistics, 12:132-157.

ALMEIDA, M. 2003. Sociolingüística. Universidad La Laguna, Tenerife.

ALMEIDA, M. y M. DÍAZ 1998. Aspectos sociolingüísticos de un cambio gramatical: la expresión de futuro. Revista Estudios Filológicos, 33:7-22.

ALMEIDA M. y C. VIDAL 1995. Variación socioestilística del léxico: un estudio contrastivo. Boletín de Filología 35:49-65

ALVAR, M. 1986. Hombre, etnia, Estado: Actitudes lingüísticas en Hispanoamérica. Gredos, Madrid.

ARAYA, G. 1973. Atlas Lingüístico- Etnográfico del Sur de Chile (ALESUCH). Editorial Universitaria, Valdivia.

BLAS, J. 1996. De nuevo el español y el catalán juntos y en contraste. Estudio de actitudes lingüísticas. Revista de Lingüística Teórica y Aplicada 34:49-62.

BLAS, J. 1999. Las actitudes hacia la variación intradialectal en la sociolingüística hispánica. Revista Estudios Filológicos 34: 47-72.

COLOANE, F. 1956. Tierra del fuego. Zig-Zag, Santiago de Chile. 1983. Cabo de hornos. Andrés Bello, Santiago de Chile. 2002. El último grumete de la Baquedano. Zig-Zag, Santiago de Chile.

DEMONTE, V. 2000. Gramática, variación y norma: una tipología. En: Estudios Hispánicos: Asociación Coreana de Hispanistas 12.

FASOLD, R. 1984. The sociolinguistics of Society. Basil Blackwell. Ltda., Oxford.

GUITARTE, G. 1991. Siete estudios sobre el español de América. Universidad Nacional Autónoma de México, DF.

HUDSON, R.1981. La Sociolingüística. Anagrama, Barcelona. KANY, CH. 1970. Sintaxis Hispanoamericana. Gredos, Madrid. 
LABOV, W. 1983. Los modelos sociolingüísticos. Cátedra, Madrid.

LÓPEZ MORALES, H. 1989. Sociolingüística. Gredos, Madrid. 1994. Métodos de investigación lingüística. Colegio de España, Salamanca.

MAKUC, M. 1999. El español de Punta Arenas, un enfoque sociolingüístico. Revista Austro Universitaria 11.

2008. Un caso de variación sintáctico-semántica del verbo en el español de Magallanes: una aproximación sociolingüística, Magallania, 36(2):79-90. Punta Arenas

2010a. Caracterización de la comunidad de habla de Magallanes: elementos lingüísticos y de identidad cultural. En Identidad regional y desarrollo para Magallanes. Universidad de Magallanes, Punta Arenas.

2010b. Los mensajes para el campo: elemento comunicativo identitario de la comunidad de habla magallánica. Magallania 38(1):97-100.

MARTINIC, M. 2006. Historia de la región magallánica. Ediciones de la Universidad de Magallanes, Punta Arenas.
MARTINIC, M. y C. BURATOVIC 2007. Las comunicaciones a distancia en Magallanes. Ediciones La Prensa Austral, Chile.

MENDIKOETXEA, A. 1999. Construcciones con se: medias, pasivas e impersonales. En: Gramática descriptiva de la Lengua Española, Bosque \& Demonte editores. Espasa Calpe, Madrid. pp. 1635-1722.

MIHOVILOVIC, N. 1974. Entre el cielo y el silencio. Pineda libros, Santiago de Chile.

MORENO, M. 2007. Colonia, los ojos de la memoria. Ivan Alcaino Mora, Punta Arenas.

ORTEGA, G.1981. El español hablado en Canarias: visión sociolingüística. Revista de Filología de la Universidad de La Laguna 0: 111-115.

OROZ, R. 1965. La lengua castellana en Chile. Facultad de Filosofía y Educación, Santiago.

SILVA, C. 1989. Sociolingüística, Teoría y análisis. Alhambra, Madrid.

VEGA, C. 2001. El trovador de la Patagonia. ATELI, Punta Arenas.

WEGMANN, O. 1976. La última canoa. Hersaprint (tomo I), Punta Arenas. 
